\title{
Study of Measurement Errors in a Current Sensor with Air-Gaps
}

\author{
A. Usón ${ }^{1}$, Letosa J. ${ }^{1}$, Arcega F.J. ${ }^{1}$, Samplón M. ${ }^{1}$, Artal J.S. ${ }^{1}$ \\ ${ }^{1}$ Department of Electrical Engineering \\ Escuela Universitaria de Ingeniería Técnica Industrial de Zaragoza (E U I T I Z.), University of Zaragoza \\ Rio Ebro Campus - María de Luna, n 3 Ed. “Torres Quevedo”, 50018 Zaragoza (Spain) \\ phone:+34 976 762589, fax:+34 976 762226, e-mail: auson@unizar.es, arcegafj@unizar.es, jletosa@unizar.es
}

\begin{abstract}
A modelization of a toroidal current sensor with air gaps using the 3D finite element method is presented. The errors associated with this sensor have been evaluated and the experimental verification has been carried out.

With this model we try to study the sensitivity to the crosstalk error of this kind of current sensors in function of two design parameters, the width of the air gap and the position of the sensor with respect to the cable carrying current.
\end{abstract}

Key words: high current, sensor modelling, finite elements analysis, measurement error, air gaps.

\section{Introduction}

The great increase in the performance of simulation tools over the last years allows today a more realistic description of the behaviour of current sensors.

The numerical techniques let us to take into account effects like fringing and leakage fields, non linearity of magnetic materials, sensitivity to crosstalk and others in a more accurate way than with the conventional analytical methods.

To simulate the behaviour of the sensor analysed in this work we use the finite element method in 3D by means of the commercial software OPERA of Vector Fields.

The current sensors with electrical insulation (current transformer and many other systems) are extensively used in high voltage and high current installations for measurement and protection purposes. Usually it is more practical a clamp-type current sensor but its accuracy is limited by the uncertainty in the gap's width. In other applications air gaps are required to avoid core saturation or to preserve linearity.

In this work, a toroidal shape current sensor with air gaps is analysed. The magnetic flux distribution has been modelled and measured inside the core, for different width of the air-gap. The sensitivity of the sensor to crosstalk errors due to neighbouring currents, external to the core, is investigated in function of several parameters as the width of the gap or the position of the sensor respect to the disturbing current. All the analysis has been focused in the low frequency range $(\leq 60 \mathrm{~Hz})$.

The aim of this communication is to contribute to improve the design of sensors with air-gaps and to establish the error dependence with several design parameters.

\section{State of the art}

Nowadays it exists a great interest in the study of sensors using numerical models because they reflect better than the analytical ones, the real behaviour of the devices. The experimental study of the influence of the air gap in a toroidal current sensor has been developed in [1], for the low current and frequency range.

The modelization of toroidal sensors with air-gaps using the finite element method has been studied in [2] [3], the magnetic circuit parameters have been calculated for modelling the measuring system.

The study of the influence of different kind of air gaps in the magnetic circuit in function of the frequency has been developed in [4].

In this work we present the analysis of the influence of the air gap and the relative position of the air gap within the toroidal core, in the measurement error of the current produced by currents in the vicinity of the sensor. This work continues and complements the other one published by the authors in [5].

\section{Modelling of the magnetic core}

A magnetic core of rectangular section and dimensions $R_{\text {in }}=24.5 \mathrm{~mm} ; R_{\text {out }}=38.6 \mathrm{~mm} ; h=25.4 \mathrm{~mm}$, and a wire carrying a current, centred in its axis, as shown in the Fig. 1, has been simulated and modelled with the finite element method.

In the core we have simulated air-gaps of different dimensions studying its influence in the magnetic flux within the core and in the gap, for different relative positions between the cable and the air-gap.

The magneto-static problem has been solved by simulation using a cylindrical model of radius $1 \mathrm{~m}$ and high $2 \mathrm{~m}$ with the toroidal core centred in it. The wire of infinite length has been simulated with one of $2 \mathrm{~m}$ length with the axis in the cylindrical model.

Initially a toroidal core without air-gaps and of linear magnetic material of relative permeability $\mu=210$ has been modelled. The results of this simulation have been compared with the ones obtained by analytical expressions (Biot-Savart law) for evaluating the errors in the numerical calculations 


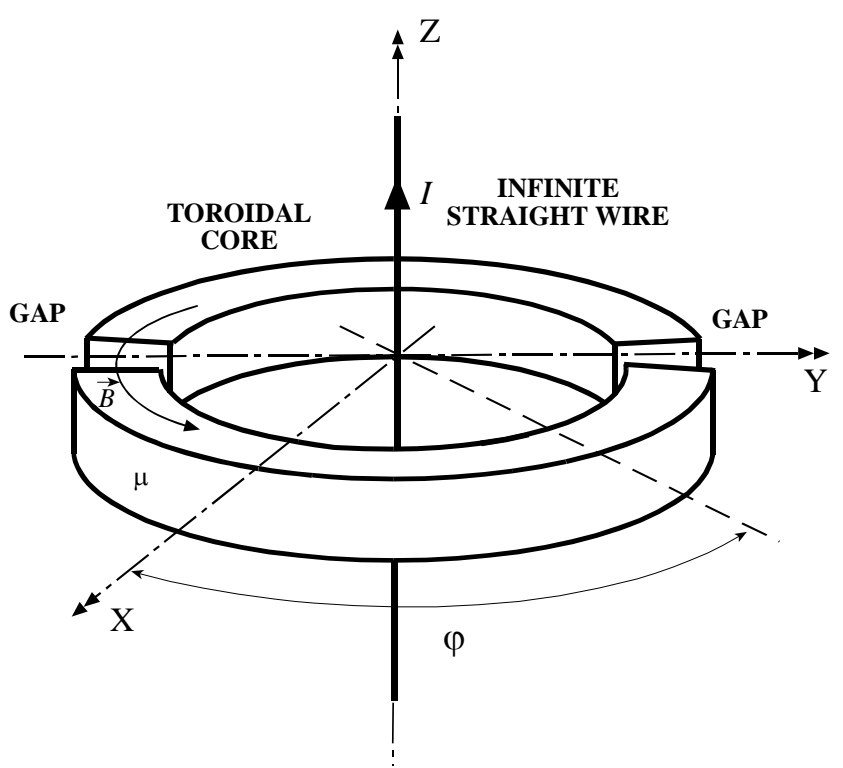

Fig.1. Torus of magnetic material with two air gaps

Errors obtained in the calculation of the magnetic field (induction) in the inner of the core are shown in Table I. In the first raw, calculated errors for several points of a circumference of average radius of the toroid are shown. In the second raw the errors for several points of a radial line in the inner of the toroid are shown.

TABLE I. Errors in the module of the magnetic flux density $\mathbf{B}$

\begin{tabular}{|c|c|c|}
\cline { 2 - 3 } \multicolumn{1}{c|}{} & $\begin{array}{c}\text { Average error } \\
\text { (\%) } \\
\text { (Biot-Savart) }\end{array}$ & $\begin{array}{c}\text { Max. error. (\%) } \\
\text { (Biot-Savart) }\end{array}$ \\
\hline $\begin{array}{c}\text { Circumference } \\
r=31,55 \mathrm{~mm}\end{array}$ & $1,58 \mathrm{E}-02$ & $5,68 \mathrm{E}-02$ \\
\hline $\begin{array}{c}\text { Radial line from } \\
r=25 \mathrm{~mm} \text { to } \\
r=38 \mathrm{~mm}\end{array}$ & $2,93 \mathrm{E}-02$ & $8,84 \mathrm{E}-02$ \\
\hline
\end{tabular}

The average error in percentage with respect to the analytical solution for the magnetic flux $\left(\Phi_{B}\right)$ calculation in transversal sections of the core is $8,28 \mathrm{E}-2$.

Later, different simulations with the non-linear curve corresponding to the behaviour of the magnetic material (standard Yellow-White) have been made. We have chosen such material because we have several samples and we can obtain experimental values.

The material characterisation has been carried out in our Electrical Laboratory (LME, accredited by ENAC, the Spanish Accreditation System) and the results are in good accordance with the ones indicated in the specifications of the manufacturer (Micrometals)

In those simulations we have introduced air gaps of different length, studying the variation of the magnetic flux in each case by simulation and by experimental measurement. The results are shown in the next paragraph

\section{Experimental results}

Three models including two symmetric air gaps of length $\ell=0,8 \mathrm{~mm}, \quad \ell=1,6 \mathrm{~mm}$ y $\ell=2,4 \mathrm{~mm}$ have been developed in the non-lineal model presented in the paragraph above and corresponding to the Yellow-White material.

The air-gaps are situated in the axis $\mathrm{YY}^{\prime}$ (see Fig. 1)

The models have been considered symmetrical respect to the plane $\mathrm{OXZ}$, in order to avoid the need of imposing the boundary conditions in the surfaces contained in the air-gaps.

The simulated values show a good accordance with the expected ones. In Fig. 2 we can see the distribution of the magnetic flux density $\mathbf{B}$ in the air-gaps. As we can appreciate, the magnetic induction vector weakens and distorts in the air-gap, producing effects of dispersion that affect the flux distribution along the whole magnetic core.

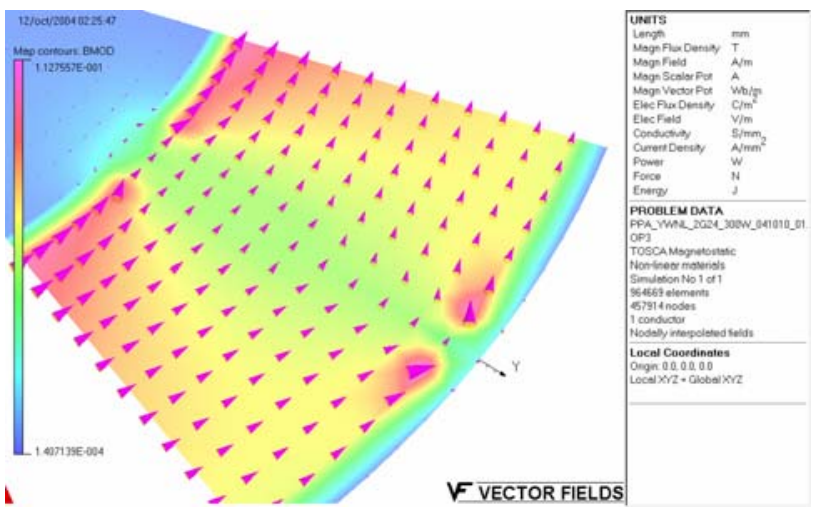

Fig. 2. Magnetic flux density and lines of $\mathbf{B}$ in the air gap of $2,4 \mathrm{~mm}$ y $300 \mathrm{~A}$

In the Fig. 3 we can appreciate the distribution of the magnetic flux in the points of a circumference of the average radius of the toroidal core in function of the position. It must be taken into account that, as specified in the Fig. 1, the angle is referred to the $X$ axis, so the air gaps are placed at 90 and 270 degrees.

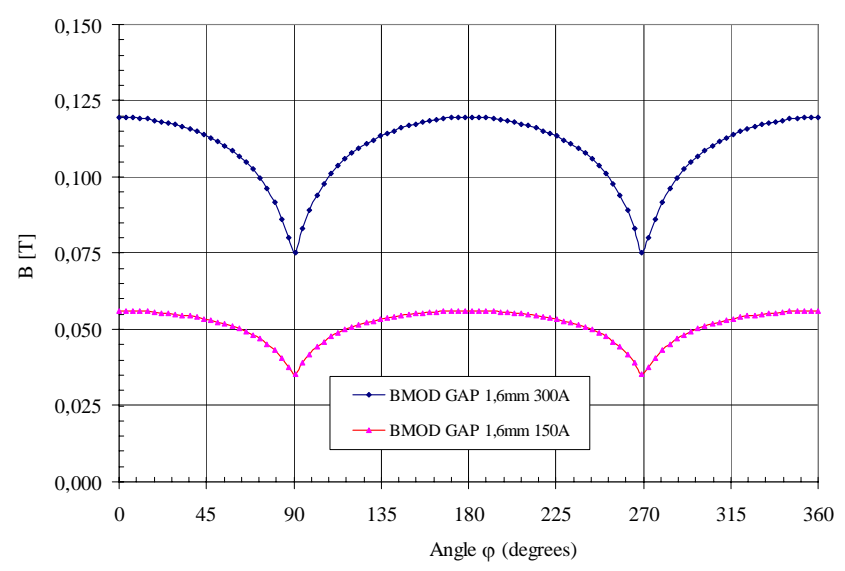

Fig. 3. Distribution of $\mathbf{B}$ module with respect to the circumference of $r=31,55 \mathrm{~mm}$. Gap of 1,6 mm

For verifying quantitatively the accuracy of the simulated data, a series of measurements have been carried out. 
For the experimental results, a toroidal core cut by its half and separated by non magnetic material (of a width as indicated in the anterior paragraph) was used.

The pick-up coil for sense the magnetic flux was built with 200 turns distributed along an angle of $3^{\circ}$ and situated at $90^{\circ}$ of the air gap $(\varphi=0)$. All the measurements were carried out for alternative current of $50 \mathrm{~Hz}$ flowing by the central wire through the torus and for two values of current of $150 \mathrm{~A}$ and $300 \mathrm{~A}$.

The results are summarized in Table II (300 A) and Table III (150 A) and are shown graphically in Fig. 4.

TABLE II. Comparison of numerical values, $\Phi_{B}$ (sim), and experimental ones, $\Phi_{B}$ (lab), of the magnetic flux for $I=300 \mathrm{~A}$

\begin{tabular}{|l|c|c|c|}
\hline$I=300 \mathrm{~A}$ & $\Phi_{B}(\operatorname{sim})[\mu \mathrm{Wb}]$ & $\Phi_{B}(\mathrm{lab})[\mu \mathrm{Wb}]$ & Error (\%) \\
\hline$\ell=0 \mathrm{~mm}$ & 138,96 & 139,31 & $-0,25$ \\
\hline$\ell=0,8 \mathrm{~mm}$ & 57,83 & 58,30 & $-0,80$ \\
\hline$\ell=1,6 \mathrm{~mm}$ & 43,23 & 42,41 & 1,92 \\
\hline$\ell=2,6 \mathrm{~mm}$ & 35,92 & 35,52 & 1,14 \\
\hline
\end{tabular}

TABLE III. Comparison of numerical values, $\Phi_{B}$ (sim), and experimental ones, $\Phi_{B}$ (lab), of the magnetic flux for $I=150 \mathrm{~A}$

\begin{tabular}{|l|c|c|c|}
\hline$I=150 \mathrm{~A}$ & $\Phi_{B}(\operatorname{sim})[\mu \mathrm{Wb}]$ & $\Phi_{B}(\mathrm{lab})[\mu \mathrm{Wb}]$ & Error (\%) \\
\hline$\ell=0 \mathrm{~mm}$ & 60,38 & 64,11 & $-5,82$ \\
\hline$\ell=0,8 \mathrm{~mm}$ & 26,59 & 27,12 & $-1,93$ \\
\hline$\ell=1,6 \mathrm{~mm}$ & 20,25 & 20,08 & 0,85 \\
\hline$\ell=2,6 \mathrm{~mm}$ & 17,03 & 16,99 & 0,23 \\
\hline
\end{tabular}

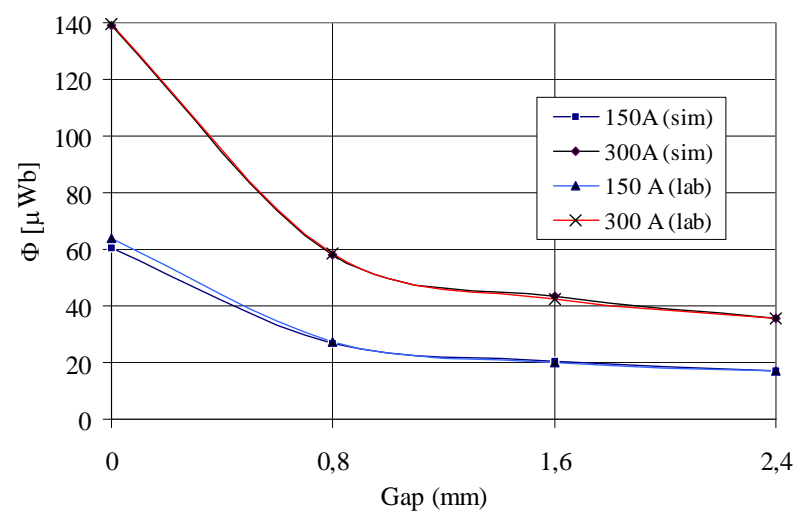

Fig.4. Magnetic flux obtained numerically and measured in the laboratory.

In Fig. 4 it can be seen the accordance between experimental data and simulated data, so that we can validate our model. After the analysis of the procedure of the measurement we can conclude that the differences between experimental and simulated values are due to uncertainties in the mechanical positioning of our experimental system.

\section{Discussion}

After comparison between analytical results and simulated ones in simple situations (without air-gap) we have established an average error in the calculation of the magnetic flux of $0,08 \%$. In consequence, the used model has enough accuracy for obtaining good predictions in the range of currents used in industrial applications.

To carry out a first verification in a real case, we have simulated a toroidal core with two symmetric air gaps of different lengths. We have calculated the mean magnetic flux along the torus cross-sections by simulation and the comparison with the experimental results measured in the laboratory has given a very good approximation as we have shown previously.

\subsection{Crosstalk errors in each section of the toroidal core} without air-gaps.

The first study is focused in the deviation of magnetic flux values in each section of a torus without air-gaps due to the external current. Fig. 5 shows the position of the external wire respect to the cross-sections of the toroidal core. Results for a torus with non-linear magnetic material (yellow-white) are shown in Fig. 6. External current was established at $300 \mathrm{~A}$ and the current flowing through the inner wire at $15 \mathrm{~A}$ (5\% of the external current). Although magnetic flux can be negative in some of the sections (the magnetic flux density vector $\mathbf{B}$ change its direction along inner lines of the torus), only absolute values have been considered due to the fact that the present study is only considering sensors based on the Faraday's induction law and the pick-up coils of the instruments are supposed uniformly distributed along the surface of the magnetic core. $\Phi_{R E F}$ stands for the magnetic flux without external current, and $\Phi$ is the total flux due to the external and the internal currents. The results depicted are related to a position $r$ of the external current two, three and four times the inner radius of the core, $R_{\text {in }}$. Smallest differences appear at sections between $\pm 20^{\circ}<\varphi< \pm 40^{\circ}$ (depending on the distance of the external current) and the biggest deviations appear always at section $\varphi=180^{\circ}$.

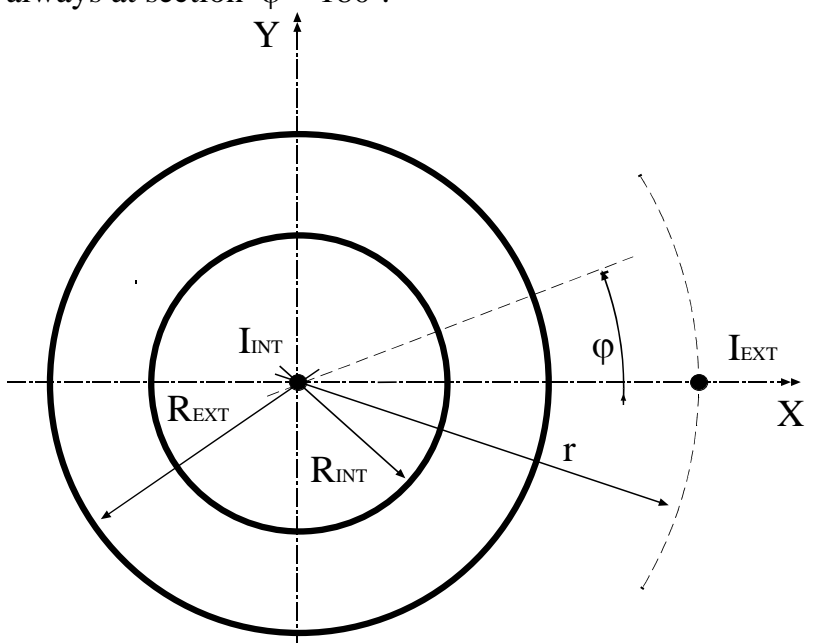

Fig.5. Schematic of the toroidal core and position of external current 


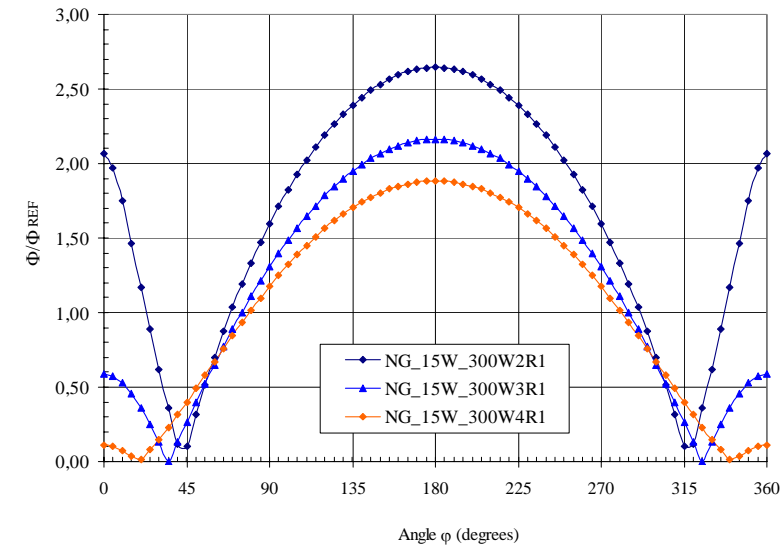

Fig.6. Magnetic flux deviations in cross-sections of the magnetic core due to a external current of $300 \mathrm{~A}$ parallel to the 15 A inner current and placed at two, three and four times $R_{\text {in }}$

5.2. Crosstalk errors in the toroidal core with two symmetrical air-gaps.

Considering instruments with pick-up coils wound uniformly along the whole magnetic core, we have estimated the crosstalk errors for three air-gaps widths and three positions of the air-gaps with respect to the external current. As shown in Fig. 5, FI $=0$ stands for a position of the two symmetrical gaps at $\varphi=0^{\circ}$ and $\varphi=180^{\circ}, \mathrm{FI}=45$ stands for a position of the two symmetrical gaps at $\varphi=45^{\circ}$ and $\varphi=225^{\circ}$ and FI $=90$ stands for a position of the two symmetrical gaps at $\varphi=90^{\circ}$ and $\varphi=270^{\circ}$. Results from the numerical simulations are shown in Table IV. The crosstalk errors are calculated using the relation

$$
\varepsilon=\frac{\Phi_{T}-\Phi_{R E F}}{\Phi_{R E F}}
$$

where $\Phi_{T}$ is the total average magnetic flux due to the internal and external currents for the gaped core and $\Phi_{R E F}$ is the average magnetic flux for the same gaped core due only to the internal current.

TABLE IV. Crosstalk errors for different air-gaps widths and positions. Internal current of $15 \mathrm{~A}$ and external current of $300 \mathrm{~A}$

\begin{tabular}{|c|c|c|c|}
\hline \multicolumn{4}{|c|}{ Crosstalk error (\%) } \\
\hline $\mathrm{FI}=0$ & $2 \times 0,8 \mathrm{~mm}$ & $2 \times 1,6 \mathrm{~mm}$ & $2 \times 2,4 \mathrm{~mm}$ \\
\hline & & & \\
\hline$r=2^{*} R_{\text {in }}$ & 55,43 & 87,93 & 117,05 \\
\hline$r=3^{*} R_{\text {in }}$ & 19,98 & 31,45 & 41,48 \\
\hline$r=4^{*} R_{\text {in }}$ & 10,08 & 16,66 & 21,85 \\
\hline & & & \\
\hline $\mathrm{FI}=90$ & $2 \times 0,8 \mathrm{~mm}$ & $2 \times 1,6 \mathrm{~mm}$ & $2 \times 2,4 \mathrm{~mm}$ \\
\hline & & & \\
\hline$r=2^{*} R_{\text {in }}$ & $-8,99$ & $-17,56$ & $-24,87$ \\
\hline$r=3^{*} R_{\text {in }}$ & $-0,58$ & $-2,58$ & $-4,72$ \\
\hline$r=4^{*} R_{\text {in }}$ & $-0,13$ & $-0,43$ & $-1,45$ \\
\hline & & & \\
\hline $\mathrm{FI}=180$ & $2 \times 0,8 \mathrm{~mm}$ & $2 \times 1,6 \mathrm{~mm}$ & $2 \times 2,4 \mathrm{~mm}$ \\
\hline & & & \\
\hline$r=2^{*} R_{\text {in }}$ & $-28,90$ & $-55,66$ & $-77,02$ \\
\hline$r=3^{*} R_{\text {in }}$ & $-11,01$ & $-23,57$ & $-33,95$ \\
\hline$r=4^{*} R_{\text {in }}$ & $-4,74$ & $-13,01$ & $-18,78$ \\
\hline
\end{tabular}

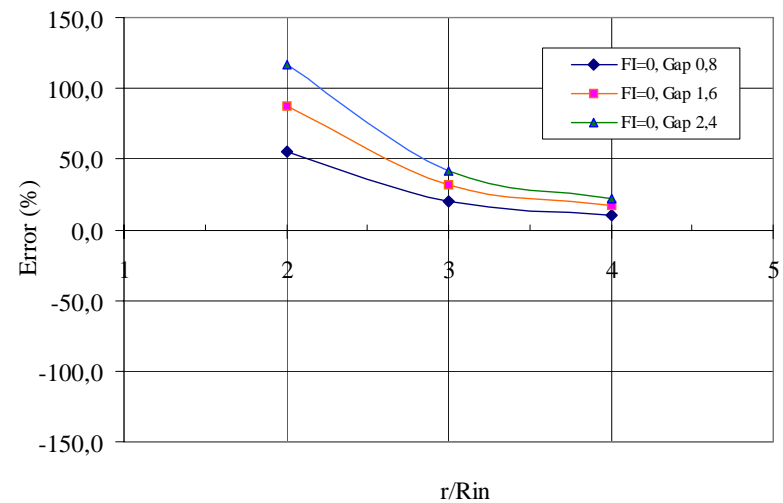

Fig.7. Crosstalk errors due to a 300 A current flowing parallel to the measured current of $15 \mathrm{~A}$ and placed outside the magnetic gaped core at two, three, and four times the inner radius $R_{i n}$ of the magnetic core. The gaps are located at $\varphi=0^{\circ}$ and $\varphi=180^{\circ}$

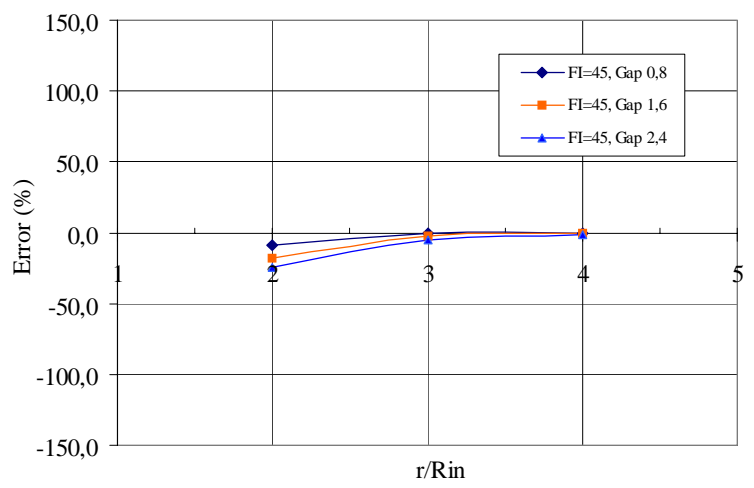

Fig.8. Crosstalk errors due to a 300 A current flowing parallel to the measured current of $15 \mathrm{~A}$ and placed outside the magnetic gaped core at two, three, and four times the inner radius $R_{i n}$ of the magnetic core. The gaps are located at $\varphi=45^{\circ}$ and $\varphi=225^{\circ}$

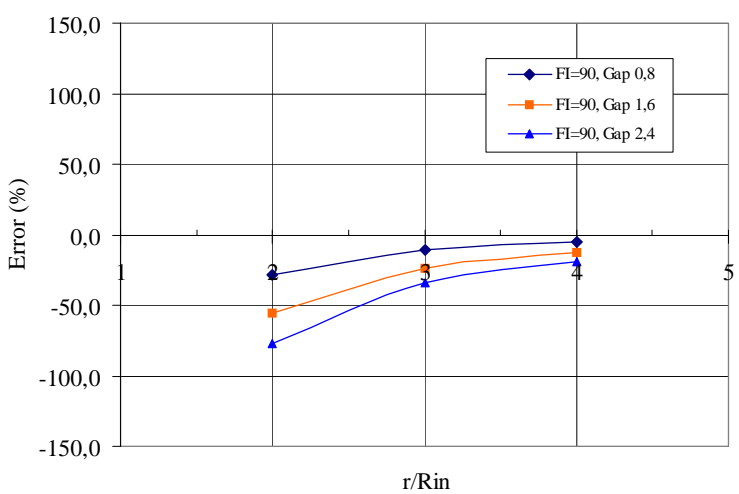

Fig.9. Crosstalk errors due to a $300 \mathrm{~A}$ current flowing parallel to the measured current of $15 \mathrm{~A}$ and placed outside the magnetic gaped core at two, three, and four times the inner radius $R_{\text {in }}$ of the magnetic core. The gaps are located at $\varphi=90^{\circ}$ and $\varphi=270^{\circ}$

In Fig. 7, Fig. 8, and Fig. 9, the numerical results are depicted in order to be more easily compared and analysed. Besides the expected decline of the crosstalk 
error when the external current separates from the magnetic core, there are two results which are interesting to underline:

- $\quad$ it is clearly seen that the crosstalk error is extremely sensitive to the position of the air-gap with respect to the external wire, and there shall be a unique position which minimise the error.

- $\quad$ The wider is the air-gap the greater are the crosstalk errors, for any position of the gap.

\section{Conclusion}

We have developed a model for calculate by the finite element method in 3D the magnetic field in the cross-sections of a toroidal type current sensor with air gaps with enough accuracy for the measurement of currents in industrial applications.

The goodness of the simulated results has been verified by analytical methods and by measurements.

Crosstalk errors due to external currents have been analysed. It has been shown that both the width of the air-gap and the position of the air-gap with respect to the external wire are key design factors when trying to minimise the influence of external current in current transformer or clamp-type current meters.

\section{Acknowledgement}

We want to thanks the University of Zaragoza, IBERCAJA and the Diputación General of Aragon (Consejeria de Ciencia, Tecnología y Universidad) for their help in our research

\section{References}

[1] Irigoyen A., Gómez-Polo C., Pérez-Conde J., "New current transformer device based on non-magnetostrictive amorphous ribbons”, Sensors and Actuators A, Vol. 91, pp. 76-79, 2001.

[2] Ducharne B., Masson J.P., Scoretti R., Lefebvre B., "Modelling of a current sensor", International Journal of Applied Electromagnetics and Mechanics, Vol. 18, pp. 14, 2003.

[3] Poulichet P., Costa F., "High-Frequency Modelling of a Current Transformer by Finite-Element Simulation"”, IEEE Transactions on Magnetics, Vol. 39, NO. 2, March 2003, pp. 998-1007.

[4] Kutkut N.H., Divan D.M., "Optimal air-gap design in high-frequency foil windings”, IEEE Trans. On Power Electronics, Vol. 13, No. 5, pp. 942-949, September 1998.

[5] Letosa J., Usón A., Artal J.S., Samplón M., Arcega F.J., "Uncertainties associated with current measurements: Analysis by finite element method", International Journal of Applied Electromagnetics and Mechanics, Vol. 19, pp. 159-164, 2004. 